\title{
Clinical and Laboratory Profile of COVID-19 Pneumonia Patients With a Complicated Post-Intensive Care Unit Hospital Course
}

\author{
Hamid Yaqoob ${ }^{a}$,, David Vernik ${ }^{b}$, Paul J. Feustel ${ }^{c}$, Dipak Chandya , Oleg Epelbaum ${ }^{\mathrm{a}}$
}

\begin{abstract}
Background: Characteristics of intensive care unit (ICU) downgrades who experience a complicated post-ICU ward course (ICU return or floor death) and the incidence of this phenomenon have not been examined in ICU survivors of coronavirus disease 2019 (COVID-19) pneumonia. The aim of the present study was to establish the rate of a complicated post-ICU ward course among survivors of COVID-19 pneumonia and describe the associated patient, ICU management, and serum biomarker characteristics. An additional aim was to compare these parameters between those who experienced a complicated post-ICU course and those who did not.
\end{abstract}

Methods: This was a retrospective study of patients who were admitted to the ICU with COVID-19 pneumonia and were downgraded to a hospital floor at the end of their initial ICU stay. Patients were divided based on a complicated or uncomplicated post-ICU course. Groups were compared with respect to relevant clinical variables. Serum biomarker levels were compared on day of ICU exit and were trended in the days preceding the downgrade. Ward stay of patients who had a complicated course was examined for notable floor events surrounding their decompensation.

Results: Eighteen out of 99 downgraded patients (18\%) experienced a complicated post-ICU course, among them there were 14 returns (14\%) and four deaths (4\%). They had higher Charlson Comorbidity Index, higher Acute Physiology and Chronic Health Evaluation (APACHE) IV score, as well as higher D-dimer and C-reactive protein (CRP) at ICU departure. They were less likely to have received therapeutic anticoagulation and convalescent plasma during their ICU

Manuscript submitted July 6, 2021, accepted July 15, 2021

Published online October 27, 2021

aDivision of Pulmonary, Critical Care, and Sleep Medicine, Westchester Medical Center, Valhalla, NY, USA

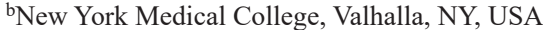

'Department of Neuroscience and Experimental Therapeutics, Albany Medical Center, Albany, NY, USA

${ }^{\mathrm{d}}$ Corresponding Author: Hamid Yaqoob, Division of Pulmonary, Critical Care, and Sleep Medicine, Westchester Medical Center, 100 Woods Road Macy Pavilion Room 1042, Valhalla, NY 10595, USA.

Email: hamid.yaqoob@wmchealth.org

doi: https://doi.org/10.14740/jocmr4555 stay. On multivariable analysis, these parameters except D-dimer remained independently associated with a complicated course. Review of biomarker trends preceding ICU exit demonstrated an upward trajectory of D-dimer, CRP, and lactate dehydrogenase (LDH) in the complicated course group not mirrored by the uncomplicated course group. Examination of notable floor events leading up to decompensation revealed that in $50 \%$ the ward course was characterized by new cardiac disturbances.

Conclusions: Our rate of ward death among ICU downgrades was similar to pre-COVID data, but the rate of ICU return was higher. Complicated post-ICU course patients were exhibiting upward biomarker trends at ICU exit, and their ward stay was punctuated by acute cardiac abnormalities.

Keywords: Hospital course; Complicated; Readmission; Intensive care unit; COVID-19; Coronavirus

\section{Introduction}

There is no paucity of studies reporting the patient characteristics, risk factors, and clinical predictors of readmission to the intensive care unit (ICU) following transfer to the hospital ward [1-9]. Some of these studies limited their primary endpoint to readmissions [1-5], while others also examined death on the ward following downgrade as an alternative metric of an unsuccessful ICU departure [6-9]. The applicability of existing studies on this subject to the conditions of the ongoing severe acute respiratory syndrome coronavirus-2 (SARS$\mathrm{CoV}-2$ ) pandemic is limited by two factors. The obvious one is that none of them included patients with novel coronavirus disease 2019 (COVID-19). The other is that very few focused exclusively on the medical ICU population, the category to which critically ill COVID-19 patients with pneumonia invariably belong. While treating critically ill COVID-19 patients in the first United States epicenter in the New York City area, it was our impression during the early months of the SARSCoV-2 pandemic that the post-ICU hospital course of those who survived to initial ICU departure was frequently punctuated by return to the ICU or unexpected death on the ward. To test this observation, we undertook the current analysis to measure the rate of ICU return or sudden ward death (compli- 
cated post-ICU course) in a cohort of critically ill patients with COVID-19 pneumonia. We also performed a comparison of the demographical, clinical, and biomarker characteristics of those who had a complicated post-ICU course to those whose hospital course following initial ICU departure did not include return to the ICU or ward death (uncomplicated post-ICU course). Finally, we explored in detail the circumstances of the complicated ward course that may have contributed to these adverse outcomes. To our knowledge, the subject of downgrade of COVID-19 pneumonia patients from the ICU has not been approached from the same perspective in any other currently published report.

\section{Materials and Methods}

\section{Patients and data collection}

This retrospective, single-center study was performed at Westchester Medical Center (WMC) in Valhalla, NY, a tertiary university academic center located in the New York City area, which was the first United States COVID-19 epicenter. All patients at least 18 years of age admitted to the ICU services at WMC between March 1, 2020 and May 31, 2020 with an International Classification of Diseases, 10th revision (ICD-10) diagnosis code of U07.1 (COVID-19 virus identified) were potentially eligible for inclusion. The presence or absence of SARS-CoV-2 infection was established via reversetranscriptase polymerase chain reaction testing of nasopharyngeal swab specimens. Patients were excluded from the study population if they met any of the following criteria: 1) primary ICU diagnosis other than COVID-19 pneumonia (incidental positivity for SARS-CoV-2); 2) expiration in the ICU during initial ICU stay; and 3) direct out-of-hospital discharge at conclusion of initial ICU stay.

Those remaining after the application of the above exclusion criteria formed the final study sample. This cohort was then divided into two groups: the complicated post-ICU course group (cases) and uncomplicated post-ICU course group (controls). Belonging to the complicated course group were those patients whose initial departure from the ICU was followed by either a return to the ICU or death on the ward before an opportunity to return to the ICU during the index hospitalization. The uncomplicated course group consisted of patients whose departure from the ICU was followed by hospital discharge without a return to the ICU during the remainder of the index hospitalization. WMC did not have a dedicated step-down unit option for ICU downgrades during the study period, so patients were transferred from ICU to floors with a nurse to patient ratio ranging from 1:6 to 1:8. Patients were managed by trainees under attending physician supervision in all settings. ICU triage decisions for both acceptance and downgrade were not protocolized but rather left to the discretion of the ICU staff. Data on demographics, comorbidities, critical illness characteristics, and COVID-19 pharmacotherapies were extracted from the institutional electronic medical record and compared between the two groups. Chronic disease burden was summarized using the Charlson Comorbidity Index (CCI)
[10]; critical illness severity was measured by the Acute Physiology and Chronic Health Evaluation (APACHE) IV score [11]. Acute kidney injury (AKI) was identified according to the criteria contained in the Kidney Disease: Improving Global Outcomes (KDIGO) guidelines [12]. ICU length of stay was based only on time in the WMC ICU and did not include time that transferred patients may have spent in outlying hospital ICUs. Therapeutic decisions in COVID-19 patients (e.g., therapeutic anticoagulation, convalescent plasma) during the study period were dictated by the clinical team rather than by an institutional algorithm or clinical trial considerations.

Also obtained were serum levels of four biomarkers commonly tracked in COVID-19 patients at our institution (without a formal protocol): D-dimer: normal range $<0.59 \mathrm{mg} / \mathrm{L}$; lactate dehydrogenase (LDH): normal range $125-220 \mathrm{U} / \mathrm{L}$; C-reactive protein $(\mathrm{CRP})$ : normal range $<0.5 \mathrm{mg} / \mathrm{dL}$; ferritin: normal range $18-370 \mu \mathrm{g} / \mathrm{L}$.

Levels were recorded at each of three time points: 2 days prior to ICU departure, 1 day prior to ICU departure, and on the day of ICU departure. These values were graphed separately for the complicated and uncomplicated course groups, and the two groups were compared with respect to these variables on the day of ICU exit.

Additionally, the post-ICU ward stay characteristics of the patients in the complicated course group were examined in detail for such elements as length of ward stay prior to death or return to ICU, significant clinical events on the ward, and circumstances of death for those who expired before return to ICU. Follow-up to hospital death or discharge for all study patients was complete by the time of data collection.

The Institutional Review Board of New York Medical College and the Clinical Research Institute of Westchester Medical Center approved this study (protocol \#14318). Due to the retrospective nature of the study, ethics committee approval and informed consent were not required.

\section{Statistical analysis}

Categorical variables are expressed as frequency (percentage) and were compared using Fisher's exact test. Continuous variables expected to be normally distributed are expressed as mean \pm standard deviation (SD) and were compared using the independent-sample $t$-test (two-tailed alpha of 0.05). Continuous variables expected to violate normality are expressed as median (interquartile range (IQR): $25-75$ th percentile), and statistical significance was assessed by the Mann-Whitney U nonparametric test. P-value of $<0.05$ was accepted as statistically significant.

Continuous and categorical variables that were statistically significantly different between the two study groups based on descriptive statistics were subjected to univariate binary logistic regression with the possible outcomes being a complicated or uncomplicated post-ICU course. All of the covariates included in the univariate analysis were then entered into a multivariable binary logistic regression model. Imputation for missing values was achieved by mean substitution. Results of the univariate and multivariable regression analysis were expressed as unadjusted and adjusted odds ratios (ORs) along 


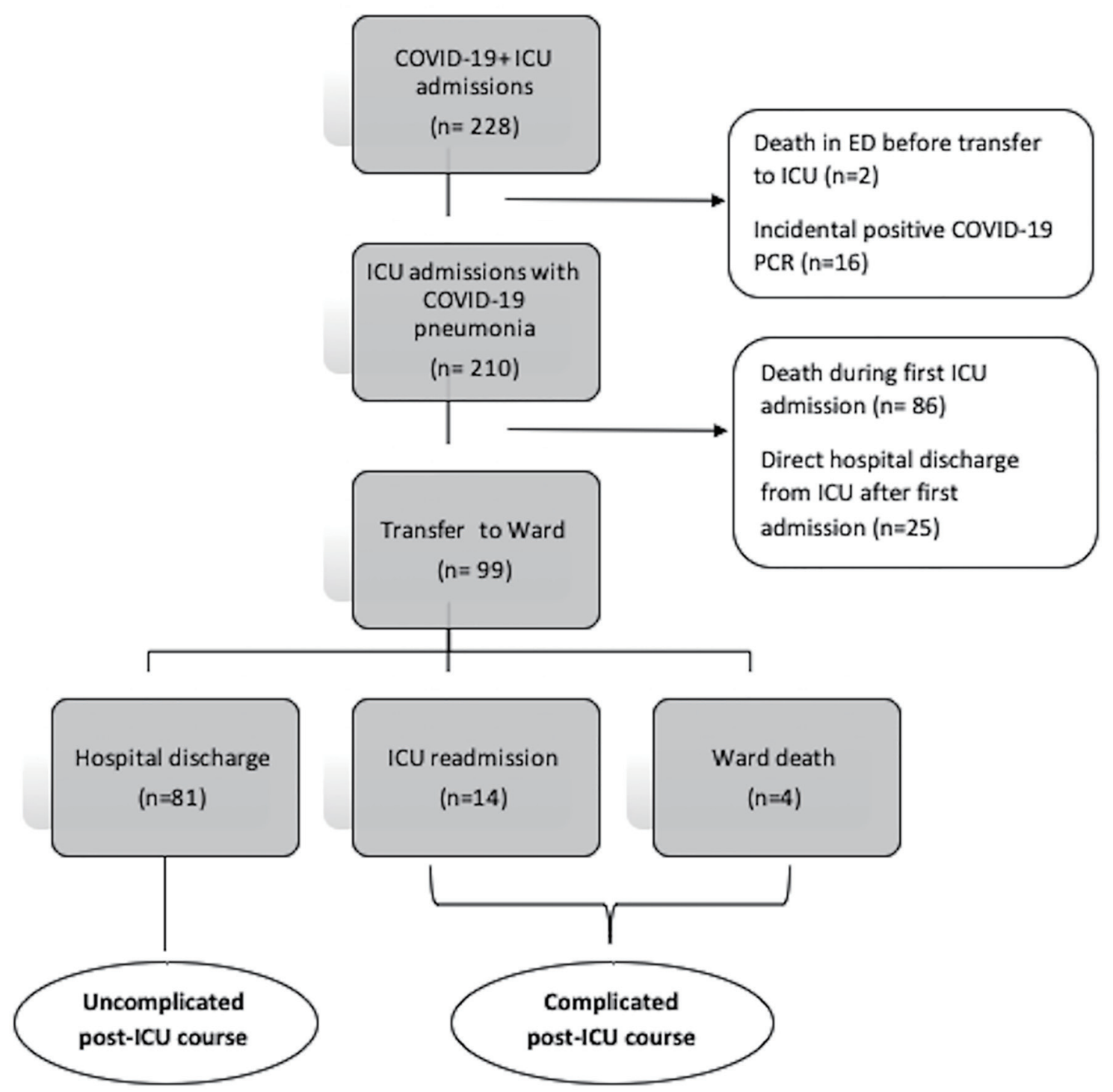

Figure 1. Flow chart depicting how the two study groups was obtained. ICU: intensive care unit; COVID-19: coronavirus disease 2019; PCR: polymerase chain reaction; ED: emergency department.

with the respective 95\% confidence intervals (CIs). All analyses were performed using the Minitab (Minitab, LLC; State College, PA) statistical software package (v.19.2020.1).

\section{Results}

The flow diagram illustrating how the study population was obtained is shown in Figure 1. A total of 228 unique COVID-19+ adults were accepted to WMC ICU services during the 3-month study period, of which 210 actually reached the ICU and were admitted primarily for COVID-19 pneumonia. Ninety-nine of these 210 patients $(47 \%)$ were downgraded to the wards at the end of their initial ICU stay. Of the 99 patients, $18(18 \%)$ experienced a complicated post-ICU hospital course as defined above, while $81(82 \%)$ had an uncomplicated post-
ICU hospital course. Subdivided further, four of the 99 downgraded patients died on the ward (4\%), and $14(14 \%)$ returned to the ICU; nine downgrades returned within 3 days of ICU exit, which constituted $9 \%$ of all downgrades (9/99) and 64\% of all who returned to the ICU (9/14). Considering only those who returned within 2 days, these numbers became 7\% (7/99) and $50 \%(7 / 14)$. Table 1 presents the demographical, historical, and clinical characteristics of the study sample, and compares the complicated course group and the uncomplicated course group with respect to these variables.

Only one patient in the study sample (uncomplicated group) was a recipient of extracorporeal membrane oxygenation. Patients with a complicated post-ICU course were significantly older $(70 \pm 11$ vs. $56 \pm 15$ years, $\mathrm{P}<0.001)$, had a significantly greater comorbidity burden (CCI $4.5 \pm 3$ vs. $2.5 \pm$ $2, \mathrm{P}=0.003)$ and had significantly higher critical illness sever- 
Table 1. Demographic and Clinical Characteristics of the Study Patients Based on Initial ICU Stay

\begin{tabular}{|c|c|c|c|}
\hline & $\begin{array}{l}\text { Patients with complicated course } \\
(n=18)\end{array}$ & $\begin{array}{l}\text { Patients with uncomplicated course } \\
(\mathrm{n}=81)\end{array}$ & P-value ${ }^{a}$ \\
\hline \multicolumn{4}{|l|}{ Demographics } \\
\hline Age, years, mean \pm SD & $70(11)$ & $56(15)$ & $<0.001 *$ \\
\hline Sex, female, n (\%) & $9(50 \%)$ & $56(69 \%)$ & 0.13 \\
\hline Sex, male, n (\%) & $9(50 \%)$ & $25(31 \%)$ & \\
\hline Smoking, $\mathrm{n}(\%)$ & & & 0.85 \\
\hline No & $12(67)$ & $56(69)$ & \\
\hline Unknown & $2(11)$ & $12(14)$ & \\
\hline \multicolumn{4}{|l|}{ Comorbidities, n (\%) } \\
\hline Obesity (BMI $\geq 30)$ & $8(44 \%)$ & $35(44 \%)$ & 0.99 \\
\hline Chronic kidney disease & $2(11 \%)$ & $10(12 \%)$ & $>0.99$ \\
\hline Stroke & $0(0 \%)$ & $1(1 \%)$ & $>0.99$ \\
\hline Diabetes mellitus & $7(39 \%)$ & $27(33 \%)$ & 0.78 \\
\hline Hypertension & $8(44 \%)$ & $33(41 \%)$ & 0.78 \\
\hline Cirrhosis & $1(6 \%)$ & $5(6 \%)$ & $>0.99$ \\
\hline Active malignancy & $0(0 \%)$ & $1(1.2 \%)$ & $>0.99$ \\
\hline History of malignancy & $3(17 \%)$ & $3(4 \%)$ & 0.11 \\
\hline Solid organ transplant & $1(6 \%)$ & $6(7 \%)$ & $>0.99$ \\
\hline Charlson Comorbidity Index, mean \pm SD & $4.5(3)$ & $2.5(2)$ & $0.003 *$ \\
\hline \multicolumn{4}{|l|}{ Admission source, $\mathrm{n}(\%)$} \\
\hline WMC ED & $6(33 \%)$ & $43(53 \%)$ & 0.13 \\
\hline Initial WMC ICU stay, days, median (IQR) & $6.5(2-10)$ & $8(3-22)$ & 0.082 \\
\hline \multicolumn{4}{|l|}{ Treatment, $\mathrm{n}(\%)$} \\
\hline Azithromycin & $16(89 \%)$ & $62(77 \%)$ & 0.35 \\
\hline Hydroxychloroquine & $16(89 \%)$ & $66(82 \%)$ & 0.73 \\
\hline Corticosteroids & $14(78 \%)$ & $59(73 \%)$ & 0.77 \\
\hline Convalescent plasma & $2(11 \%)$ & $40(49 \%)$ & $0.003 *$ \\
\hline Therapeutic anticoagulation & $6(33 \%)$ & $60(74 \%)$ & $0.002 *$ \\
\hline \multicolumn{4}{|l|}{ Biomarkers at ICU entry, median (IQR) } \\
\hline D-dimer (mg/L) & $2.1(1.3-19)$ & $2.3(0.92-4.2)$ & 0.27 \\
\hline LDH (U/L) & $515(370-643)$ & $530(374-721)$ & 0.73 \\
\hline $\mathrm{CRP}(\mathrm{mg} / \mathrm{dL})$ & $14.5(9.2-25)$ & $15(7.2-21)$ & 0.54 \\
\hline Ferritin $(\mu \mathrm{g} / \mathrm{L})$ & $963(412-1,759)$ & $1192(525-2,920)$ & 0.50 \\
\hline
\end{tabular}

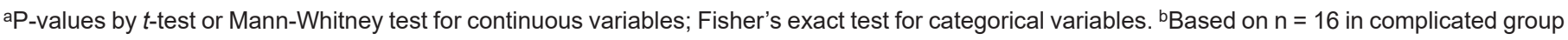
and $n=71$ in uncomplicated group. 'Based on $n=12$ in complicated group and $n=51$ in uncomplicated group. *Statistical significance with a P-value less than 0.05. AKI: acute kidney injury; APACHE: Acute Physiology and Chronic Health Evaluation; BMI: body mass index; CRP: C-reactive protein; ED: emergency department; ICU: intensive care unit; IQR: interquartile range; LDH: lactate dehydrogenase; $P F$ : $\mathrm{PaO}_{2} / \mathrm{FiO}_{2}$; RRT: renal replacement therapy; SD: standard deviation; WMC: Westchester Medical Center. 
ity on ICU entry (APACHE IV score 87 (IQR 51 - 112) vs. 57 (IQR 43 - 72), $\mathrm{P}=0.01$ ). Conversely, exposure to therapeutic anticoagulation during the initial ICU course was more common in the uncomplicated post-ICU course group: 60 patients $(75 \%)$ vs. six patients $(33 \%)(\mathrm{P}=0.002)$. The same was true of receipt of convalescent plasma: $40(49 \%)$ vs. two (11\%) in the uncomplicated and complicated groups, respectively $(\mathrm{P}=$ $0.003)$.

Figure 2 consists of line graphs representing median biomarker levels (D-dimer, LDH, CRP, and ferritin) in the two study groups as measured 2 days before ICU exit, 1 day before ICU exit, and on the day of ICU exit. Visual inspection of Figure 2 reveals a steady rise in D-dimer over the three charted days in the complicated course group, a trend not paralleled by the uncomplicated group in which D-dimer remained relatively flat. An upward trajectory on the day of ICU exit in CRP and LDH was observed in the complicated course group, whereas these biomarkers exhibited a downward trajectory in the uncomplicated course group. Ferritin, in contrast, trended down on day of ICU exit in the complicated course group while remaining flat in the uncomplicated course group. Figure 3 juxtaposes box-and-whisker plots of the median level of each of the four biomarkers on day of ICU exit in the complicated course group versus the uncomplicated course group. Median levels of all four biomarkers were numerically higher in the complicated course group compared to the uncomplicated course group, but only in the case of D-dimer and CRP (P $=0.006$ by the Mann-Whitney U-test for both) did the difference reach statistical significance. At ICU entry, there was no significant difference between the groups in median D-dimer and CRP levels (Table 1).

The results of logistic regression analysis are presented in Table 2. Age was omitted from the model because it was expected to be collinear with APACHE IV as one of its components. Increase in all included continuous variables (CCI, APACHE IV at ICU entry, D-dimer at ICU exit, and CRP at ICU exit) significantly raised the odds of a complicated post-ICU ward course in univariate analysis. On multivariable analysis, the association of D-dimer at ICU exit with this outcome lost statistical significance (adjusted OR 1.10 (95\% CI 1.00 - 1.22), P = 0.057), which was preserved for the other covariates.

Table 3 summarizes the salient details of the post-ICU ward stay that ended in either death or ICU return in the 18 patients from the complicated course group. None of these patients departed the ICU with care-limiting directives in place. The defining event occurred a median of 2.5 days (IQR 1.3 6.8 ) into the index post-ICU ward stay; the median number of days remained unchanged when calculated only for ICU readmissions. Four of the 18 patients $(22 \%)$ died on the ward, one of whom had do not intubate and do not resuscitate orders in place at time of passing. A unifying motif in the ward course of the other three patients who died was the occurrence of acute electrocardiographic disturbances. In two of them, prolongation of the QTc interval (i.e., $\geq 500 \mathrm{~ms}$ ) was detected, reaching $>600 \mathrm{~ms}$ on the most extreme measurement in one. The other 14 patients (78\%) returned to the ICU following decompensation on the ward, with $13(93 \%)$ of them requiring invasive mechanical ventilation (IMV) in the process. Of these 14, five $(36 \%)$ died during their second ICU stay. Of the nine survivors of ICU return, eight ( $89 \%)$ reached hospital discharge, all to skilled nursing facilities. One survivor of ICU return subsequently died on the ward for a hospital mortality among ICU readmissions of $6 / 14(43 \%)$. There was no statistically significant difference between survival to first ICU exit and survival to second ICU exit: $124 / 210$ (59\%) vs. 9/14 (64\%) $(\mathrm{P}=0.78)$ by Fisher's exact test. The most common ward complications noted in the 14 patients who returned to the ICU were: 1) new electrocardiographic disturbance $(29 \%) ; 2)$ new troponemia $(29 \%)$; 3) documented or suspected infection $(29 \%)$; and 4) persistent alteration of sensorium $(21 \%)$.

In aggregate, $50 \%$ of patients in the complicated course group experienced at least one of the adverse cardiac events listed in item 1 or item 2 above.

\section{Discussion}

COVID-19 survivorship studies have so far focused primarily on readmission following hospital discharge $[13,14]$. The current study, to our knowledge, is the first to report the patient characteristics, clinical parameters, and notable ward events associated with a complicated post-ICU course in the hospital, defined as ward death or return to ICU, in critically ill COVID-19 patients downgraded to the regular floor from the ICU. Supporting our hypothesis, the complicated course rate of $18 \%$ in our population was higher than similar pre-COVID numbers from mixed ICUs in France (6.6\%) [7] and a medical ICU in the United States of America (9.3\%) [1]. Noteworthy findings emerged when the composite outcome was separated into its two components. The $4 \%$ ward death rate among ICU downgrades in our study was identical to that measured in a consortium of busy US ICUs in the pre-COVID era [8]. Our ICU readmission numbers, however, turned out to be higher than the estimates derived from heterogeneous non-COVID critically ill populations. Few studies have reported such numbers among purely medical ICU patients, which would be the most telling comparison with our results. Jo et al [4] reported a $9.6 \%$ overall readmission rate in a pre-COVID South Korean study restricted to the medically critically ill, higher than typical mixed ICU range of 4-6\% [9] but still lower than the $14 \%$ in our sample. The same disparity holds true in comparison to a prior medical ICU study from the United States of America, in which $8.8 \%$ of patients were readmitted [1]. Our remarkably high ICU readmission rate may be a function of unprecedented ICU strain experienced during the study period by institutions located in the first COVID-19 epicenter in the United States of America. Under conditions of increased strain, which are a risk factor for ICU readmissions [8], the desire to maintain maximal ICU throughput could prompt premature downgrades to the ward. Corroborating this theory is the trend toward a shorter initial ICU stay in patients with a complicated postICU course. At least one prior study not limited to a highstrain environment found the opposite: a longer initial ICU stay among downgrades who subsequently returned to the ICU [2]. An additional factor to consider when juxtaposing our ICU population with COVID-19 pneumonia to prior ICU cohorts with respect to rates of ICU return is that the critically ill pa- 


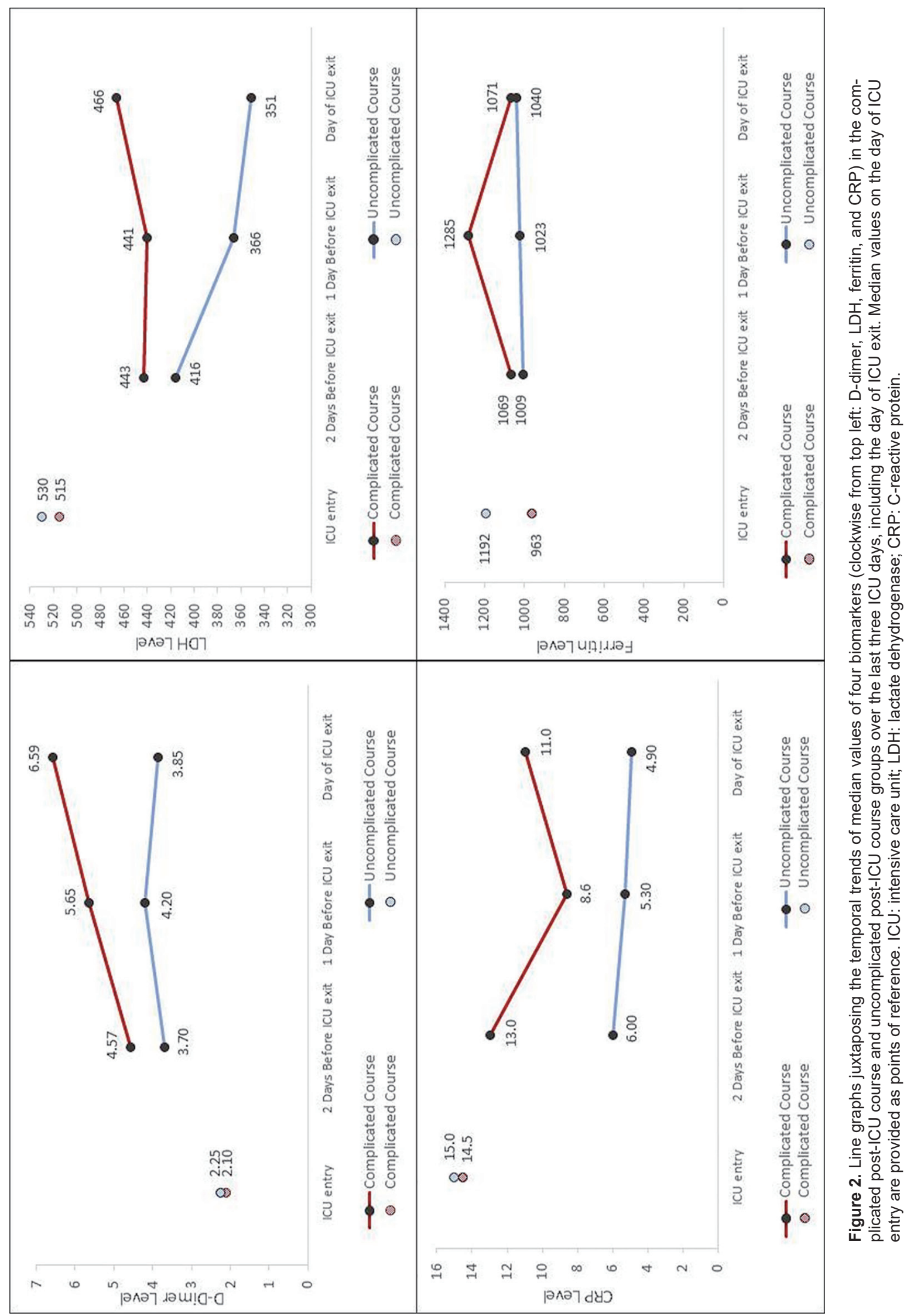



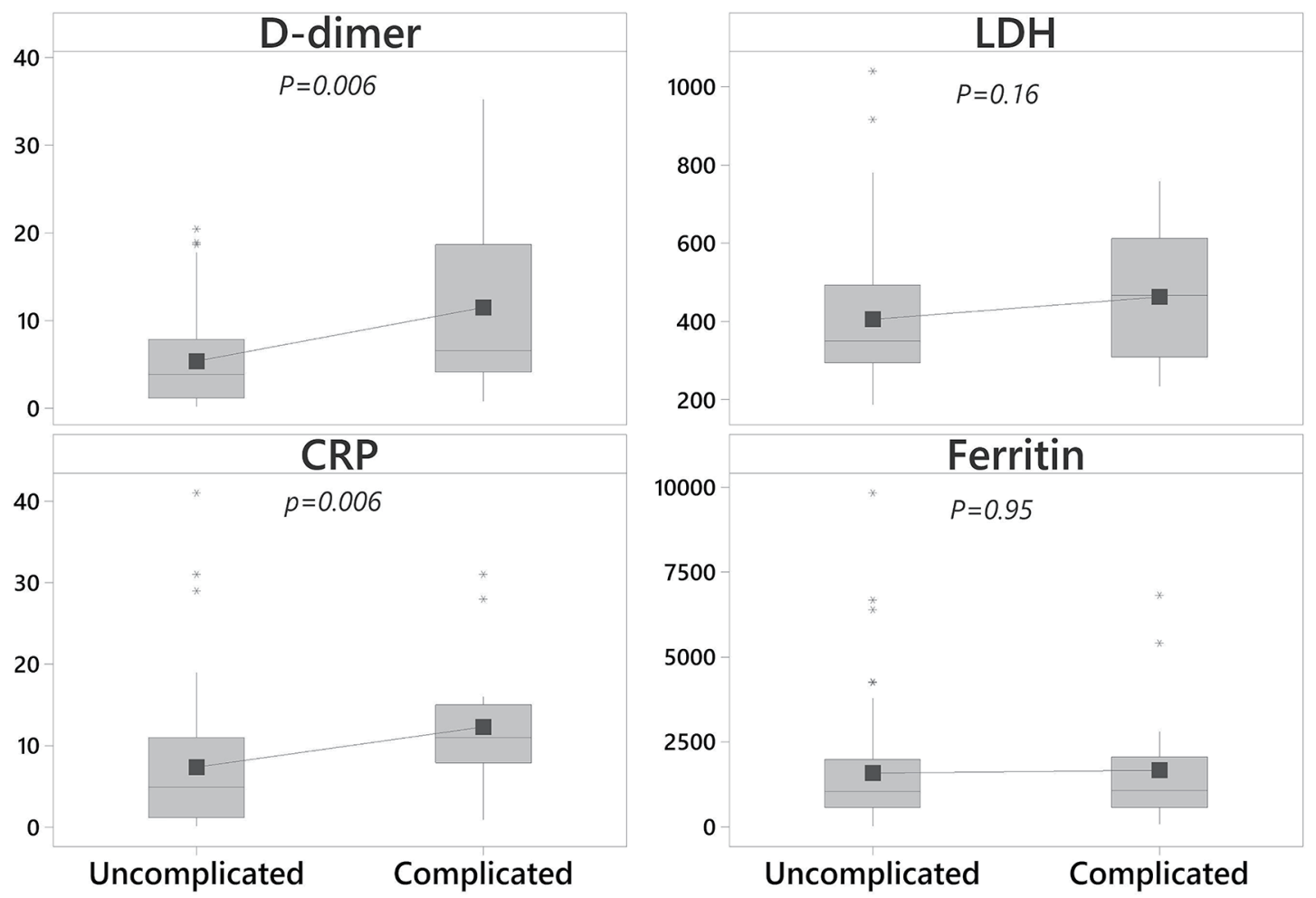

Figure 3. Tukey box plots comparing median biomarker levels in the two groups on day of ICU exit. The central rectangle spans the interquartile range (IQR), and the line within the rectangle marks the median. The square symbol marks the mean. The "whiskers" above and below the box are drawn to the furthest point within $1.5 \times$ IQR from the box (the non-outlier range). Asterisks outside the whiskers are outliers. P-values are from the Mann-Whitney nonparametric test. ICU: intensive care unit.

tients in this study all had catastrophic respiratory failure fulfilling criteria for at least moderate, and in many cases severe, acute respiratory distress syndrome at some point during their ICU course. COVID-19 pneumonia carried a 41\% mortality rate during the initial ICU stay in our population, commensurate with the magnitude of associated respiratory failure. In the study by Jo et al limited to a medical ICU contingent, the same figure was $31 \%$, whereas in mixed ICU readmission studies, this rate is quoted to be dramatically lower at approximately $7-9 \%[2,3]$.

Similar to pre-COVID studies, we found the patient factor of higher comorbidity burden and the disease severity factor of higher APACHE IV score on ICU admission to be independently associated with greater odds of a complicated postICU course. Several studies have proposed clinical prediction scores for ICU readmission $[1,6,7]$. These have tended to be cumbersome, limiting their widespread adoption. A unique feature of ICU survivors of COVID-19 pneumonia is the availability of serum biomarker levels (CRP, LDH, ferritin, and Ddimer) in the days preceding ICU exit and on day of departure that have historically not been followed with such regularity or assessed as predictors of readmission prior to COVID-19.

Table 2. Patient and ICU Factors Associated With a Complicated Post-ICU Course Based on Univariate and Multivariable Logistic Regression

\begin{tabular}{lllll}
\hline Variable & Crude OR (95\% CI) & P-value & Adjusted OR (95\% CI) & P-value \\
\hline CCI & $1.39(1.12-1.72)$ & 0.003 & $1.94(1.22-3.09)$ & 0.005 \\
APACHE IVa, b & $1.27(1.05-1.53)$ & 0.013 & $1.39(1.05-1.82)$ & 0.020 \\
D-dimerc, d & $1.11(1.03-1.19)$ & 0.008 & $1.10(1.00-1.22)$ & 0.057 \\
CRPc, e & $1.07(1.00-1.13)$ & 0.043 & $1.16(1.04-1.30)$ & 0.009 \\
Anticoagulation receipt & $0.18(0.06-0.53)$ & 0.002 & $0.07(0.01-0.50)$ & 0.008 \\
Convalescent plasma receipt & $0.13(0.03-0.59)$ & 0.009 & $0.09(0.01-0.62)$ & 0.015 \\
\hline
\end{tabular}

aValues missing for $12 / 99$ patients. ${ }^{b}$ Result from day of ICU entry. ${ }^{c}$ Result from day of ICU exit. dValues missing for 26/99 patients. ${ }^{e}$ Values missing for 24/99 patients. APACHE: Acute Physiology and Chronic Health Evaluation; CCl: Charlson Comorbidity Index; CRP: C-reactive protein; ICU: intensive care unit; OR: odds ratio; Cl: confidence interval. 
Table 3. Descriptive Summary of Notable Ward Stay Characteristics of the Patients With a Complicated Post-ICU Course

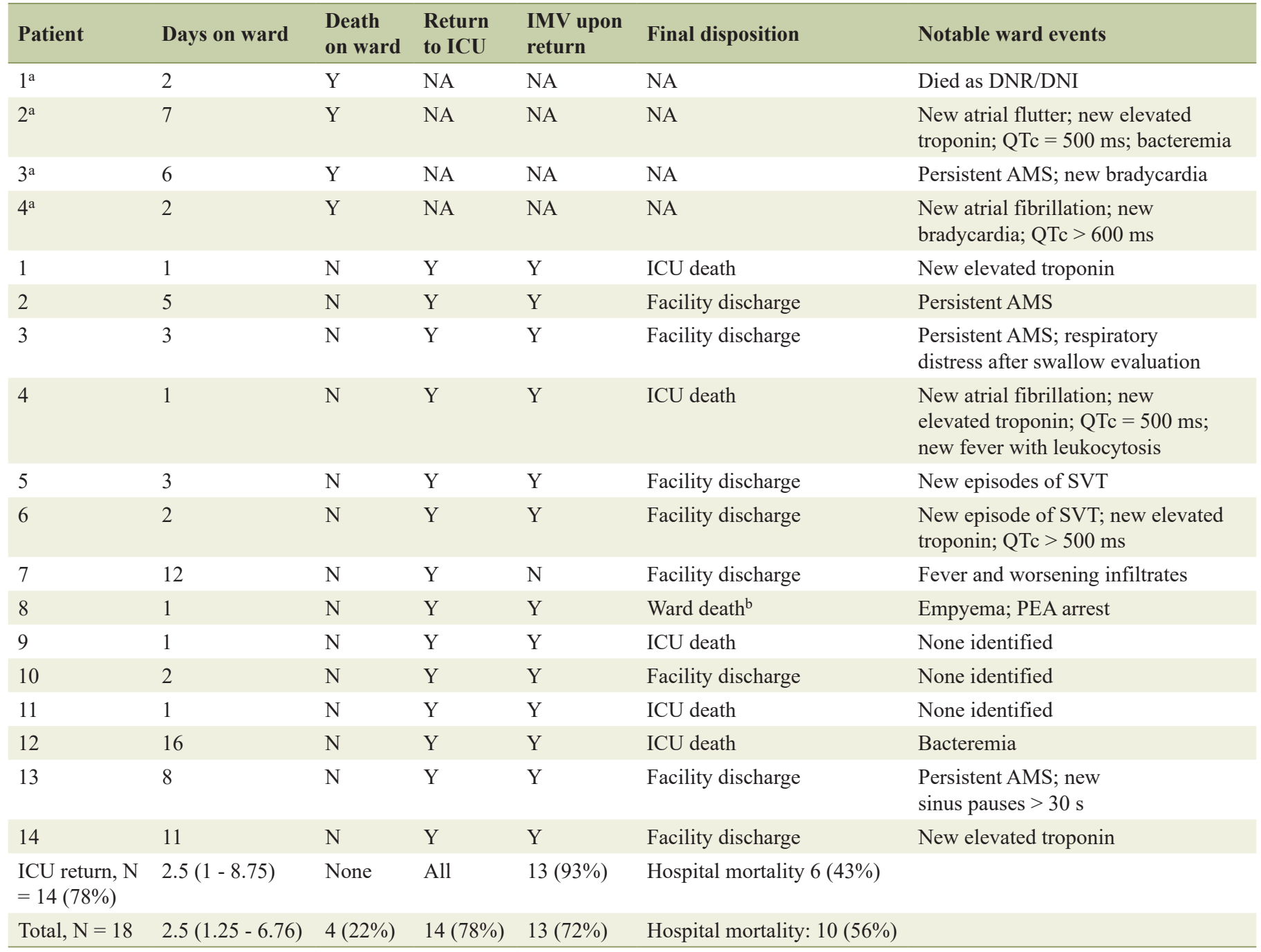

aWard deaths after initial ICU downgrade. 'batient died on the ward after repeat ICU downgrade. AMS: altered mental status; DNI: do not reintubate; DNR: do not resuscitate; ICU: intensive care unit; IMV: invasive mechanical ventilation; NA: not applicable; PEA: pulseless electrical activity; SVT: superventricular tachycardia.

Viewed retrospectively from day of ICU departure, our results demonstrate opposing trends in D-dimer and CRP levels depending on ultimate post-ICU course: an upward trajectory in patients destined for a complicated course and a downward trajectory in those destined for an uncomplicated course. While similar between the groups at ICU entry, absolute values of these two biomarkers were significantly higher on the day of ICU exit in the complicated group, though only the CRP level was independently associated with greater odds of experiencing a complicated post-ICU course after multivariable analysis. This component of our findings suggests that careful attention to the behavior of D-dimer and especially CRP levels may be warranted when COVID-19 pneumonia patients in the ICU are being considered for transfer to the ward. An upward trend in either parameter should be viewed as a cautionary sign in a candidate for ICU downgrade. Discerning the impact, if any, of evolving superimposed infection on biomarker behavior was beyond the resolving power of this study. The exact role of serum biomarkers as predictors of ICU readmission or death on the floor merits further elucidation in dedicated studies.

Close examination of ward events surrounding death or return to ICU listed in Table 3 revealed the common occurrence of acute electrocardiographic abnormalities, including instances of profoundly prolonged QTc intervals. As seen in Table 1, nearly $90 \%$ of the complicated course group received hydroxychloroquine and azithromycin, which was a standard COVID-19 treatment regimen in the early phase of the pandemic. Both medications are synergistic in causing QTc interval prolongation and thus possibly cardiac instability [15]. However, the rate of receipt of these agents was similar in the uncomplicated course group. New troponemia was another cardiac disturbance frequently detected on the ward in those with a complicated course. Troponin elevation has been well described in COVID-19 and is a negative prognostic indica- 
tor [16]. While the mechanisms of troponemia in COVID-19 remain speculative, it is intriguing to consider whether the upward trend in D-dimer and CRP levels observed in the complicated course group at ICU exit presaged a wave of hypercoagulability and hyperinflammation, respectively, destined to occur on the ward, both phenomena having been hypothesized as contributors to troponin release in COVID-19 [17]. Rather than the direct mediator of an adverse floor event, troponemia on the ward may have been a marker of an evolving proinflammatory and pro-thrombotic state that predisposed to the index event. It is also noteworthy in the same context that therapeutic anticoagulation use in the ICU was significantly less frequent in the complicated course group, a measure that could attenuate the onset of both in situ coronary thrombosis and atherosclerotic plaque thrombosis in an inflammatory milieu, especially if continued during the subsequent ward stay. The role of cardiac derangements in complicating the ward course of ICU survivors of COVID-19 pneumonia warrants separate investigation.

Our study suffers from a number of important limitations, primary among them its single-center retrospective nature and small sample size. As such, our results may have limited generalizability. Because patient data were not collected in a systematic, prospective fashion, there were missing biomarker values and cases for which APACHE IV scores at ICU entry could not be calculated. Contrary to certain pre-COVID studies of ICU readmission, our investigation did not include physiological parameters and illness severity metrics at the time of ICU departure, so this report cannot provide all-encompassing guidance for ICU downgrade decision-making in COVID-19 pneumonia. Finally, we reported the features of the post-ICU ward course of the complicated group descriptively without comparison to the uncomplicated group. This diminishes the utility of observations made on the basis of that part of the study, but a comparative analysis of ward course features between the two groups was felt to be beyond the scope of this exploratory investigation. Such analysis would be best reserved for a follow-up study with that as its primary focus.

\section{Conclusions}

The ward death rate we observed among patients with COVID-19 pneumonia downgraded from the ICU was comparable to that reported in pre-COVID medically critically ill populations, whereas the ICU readmission rate was higher than the typically reported range. Caution should be exercised when considering COVID-19 pneumonia patients with uptrending D-dimer and CRP levels for floor transfer from ICU. The ward course of ICU downgrades with COVID-19 pneumonia destined for death on the floor or ICU return is frequently punctuated by acute cardiac disturbances.

\section{Acknowledgments}

We thank Dr. Christian Becker, MD PhD for his role in the provision of APACHE IV scoring data for this project.

\section{Financial Disclosure}

None to declare.

\section{Conflict of Interest}

None to declare.

\section{Informed Consent}

Not applicable.

\section{Author Contributions}

Conceptualization and study design: Hamid Yaqoob, Dipak Chandy, and Oleg Epelbaum. Data acquisition: Hamid Yaqoob and David Vernik. Data analysis and statistics: Hamid Yaqoob, Paul Feustel, Dipak Chandy, and Oleg Epelbaum. Manuscript preparation and writing: Hamid Yaqoob and Oleg Epelbaum. Review and editing of the first draft: all authors. Approval of the final version: all authors.

\section{Data Availability}

The data supporting the findings of this study are available from the corresponding author upon reasonable request.

\section{References}

1. Gajic O, Malinchoc M, Comfere TB, Harris MR, Achouiti A, Yilmaz M, Schultz MJ, et al. The Stability and Workload Index for Transfer score predicts unplanned intensive care unit patient readmission: initial development and validation. Crit Care Med. 2008;36(3):676-682.

2. Brown SE, Ratcliffe SJ, Kahn JM, Halpern SD. The epidemiology of intensive care unit readmissions in the United States. Am J Respir Crit Care Med. 2012;185(9):955964.

3. Kramer AA, Higgins TL, Zimmerman JE. Intensive care unit readmissions in U.S. hospitals: patient characteristics, risk factors, and outcomes. Crit Care Med. 2012;40(1):310.

4. Jo YS, Lee YJ, Park JS, Yoon HI, Lee JH, Lee CT, Cho YJ. Readmission to medical intensive care units: risk factors and prediction. Yonsei Med J. 2015;56(2):543-549.

5. Liu CL, Chen WJ, Su J, Yang YH, Lin CY, Lin RL. Characteristics and outcomes of patients readmitted to the medical intensive care unit: a retrospective study in a tertiary hospital in Taiwan. Int J Gerontol. 2017;11:244-248.

6. Badawi O, Breslow MJ. Readmissions and death after ICU discharge: development and validation of two predictive models. PLoS One. 2012;7(11):e48758. 
7. Ouanes I, Schwebel C, Francais A, Bruel C, Philippart F, Vesin A, Soufir L, et al. A model to predict short-term death or readmission after intensive care unit discharge. J Crit Care. 2012;27(4):422 e421-429.

8. Wagner J, Gabler NB, Ratcliffe SJ, Brown SE, Strom BL, Halpern SD. Outcomes among patients discharged from busy intensive care units. Ann Intern Med. 2013;159(7):447-455.

9. Hosein FS, Roberts DJ, Turin TC, Zygun D, Ghali WA, Stelfox HT. A meta-analysis to derive literature-based benchmarks for readmission and hospital mortality after patient discharge from intensive care. Crit Care. 2014;18(6):715.

10. Charlson ME, Pompei P, Ales KL, MacKenzie CR. A new method of classifying prognostic comorbidity in longitudinal studies: development and validation. J Chronic Dis. 1987;40(5):373-383.

11. Zimmerman JE, KramerAA, McNair DS, Malila FM. Acute Physiology and Chronic Health Evaluation (APACHE) IV: hospital mortality assessment for today's critically ill patients. Crit Care Med. 2006;34(5):1297-1310.

12. Kellum JA, Lameire N, Kdigo Aki Guideline Work Group. Diagnosis, evaluation, and management of acute kidney injury: a KDIGO summary (Part 1). Crit Care.
2013;17(1):204.

13. Donnelly JP, Wang XQ, Iwashyna TJ, Prescott HC. Readmission and death after initial hospital discharge among patients with COVID-19 in a large multihospital system. JAMA. 2021;325(3):304-306.

14. Lavery AM, Preston LE, Ko JY, Chevinsky JR, DeSisto CL, Pennington AF, Kompaniyets L, et al. Characteristics of hospitalized COVID-19 patients discharged and experiencing same-hospital readmission - United States, March-August 2020. MMWR Morb Mortal Wkly Rep. 2020;69(45):1695-1699.

15. Mercuro NJ, Yen CF, Shim DJ, Maher TR, McCoy CM, Zimetbaum PJ, Gold HS. Risk of QT interval prolongation associated with use of hydroxychloroquine with or without concomitant azithromycin among hospitalized patients testing positive for coronavirus disease 2019 (COVID-19). JAMA Cardiol. 2020;5(9):1036-1041.

16. Maeda T, Obata R, Rizk D, Kuno T. Cardiac Injury and Outcomes of Patients With COVID-19 in New York City. Heart Lung Circ. 2021;30(6):848-853.

17. Tersalvi G, Vicenzi M, Calabretta D, Biasco L, Pedrazzini G, Winterton D. Elevated troponin in patients with coronavirus disease 2019: possible mechanisms. J Card Fail. 2020;26(6):470-475. 